\title{
Adiponectin deficiency exacerbates age-related hearing impairment
}

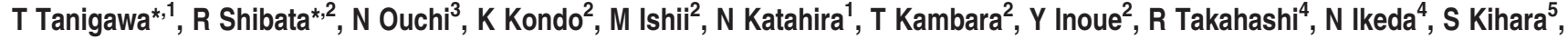 \\ H Ueda ${ }^{1}$ and T Murohara ${ }^{2}$
}

Obesity-related disorders are closely associated with the development of age-related hearing impairment (ARHI). Adiponectin (APN) exerts protective effects against obesity-related conditions including endothelial dysfunction and atherosclerosis. Here, we investigated the impact of APN on ARHI. APN-knockout (APN-KO) mice developed exacerbation of hearing impairment, particularly in the high frequency range, compared with wild-type (WT) mice. Supplementation with APN prevented the hearing impairment in APN-KO mice. At 2 months of age, the cochlear blood flow and capillary density of the stria vascularis (SV) were significantly reduced in APN-KO mice as compared with WT mice. APN-KO mice also showed a significant increase in terminal deoxynucleotidyl transferase dUTP nick end labeling (TUNEL)-positive apoptotic cells in the organ of Corti in the cochlea at 2 months of age. At the age of 6 months, hair cells were lost at the organ of Corti in APN-KO mice. In cultured auditory HEI-OC1 cells, APN reduced apoptotic activity under hypoxic conditions. Clinically, plasma APN levels were significantly lower in humans with ARHI. Multiple logistic regression analysis identified APN as a significant and independent predictor of ARHI. Our observations indicate that APN has an important role in preventing ARHI.

Cell Death and Disease (2014) 5, e1189; doi:10.1038/cddis.2014.140; published online 24 April 2014

Subject Category: Experimental Medicine

Age-related hearing impairment $(\mathrm{ARHI})$ is characterized by progressive deterioration of auditory sensitivity associated with aging. ${ }^{1}$ It is common in older populations, with an estimated prevalence of 30-60\%, and it has a strong influence on quality of life. ${ }^{2,3}$ Thus, ARHI is anticipated to become a more serious public health issue.

Recent reports demonstrated that higher body mass index (BMI) and waist circumference are implicated in the severity of hearing loss. ${ }^{4,5}$ Obesity-related disorders including dyslipidemia and type 2 diabetes are also associated with ARHI. ${ }^{6,7,8}$ Consistent with clinical observations, hyperlipidemic apolipoprotein E-knockout (ApoE-KO) mice develop hearing loss in an age-dependent manner. ${ }^{9} \mathrm{~A}$ diabetic condition generated by streptozotocin injection or a high-fat diet also leads to exacerbation of hearing loss in aged mice. ${ }^{10,11}$ In contrast, it has been shown that caloric restriction slows the progression of $\mathrm{ARHI}$ in various strains of mice. ${ }^{12,13}$ These data suggest that metabolic dysfunction under conditions of obesity is causally linked to the development of ARHI.

Adipose tissue secretes a variety of bioactive molecules, also referred to as adipocytokines, that affect obesity-linked disorders. ${ }^{14}$ APN is an adipocytokine that is downregulated in obesity-linked diseases including metabolic syndrome, type 2 diabetes, hyperlipidemia, and hypertension. ${ }^{14,15} \mathrm{We}$ and others have shown that APN-KO mice exhibit exacerbation of diet-induced insulin resistance, impaired angiogenic responses to ischemia, and impaired endothelium-dependent vasodilation. $^{16,17,18}$ Conversely, overexpression of APN promotes insulin sensitivity and angiogenesis, and reduces atherosclerotic lesion formation in a mouse model of atherosclerosis. ${ }^{17,18,19}$ These data suggest that APN functions as a mediator of obesity-related vascular and metabolic disorders. In this study, we investigated whether APN affects early sensorineural hearing loss in vivo and in vitro, and assessed its clinical significance in ARHI.

\section{Results}

Highly elevated auditory brainstem response threshold in APN-KO mice. To test whether APN affects hearing impairment, we assessed auditory brainstem response (ABR) thresholds in WT and APN-KO mice at the ages of 2 months and 6 months ( $n=5-7$ per group). WT mice did not show any threshold shifts at any frequencies at these ages. At 2 months of age, APN-KO mice exhibited a significant increase in ABR threshold at high frequency $(32 \mathrm{kHz})$ as compared with WT mice $=($ Figure $1, P<0.01)$. However, at 2 months of age APN-KO mice exhibited only slight

\footnotetext{
${ }^{1}$ Department of Otolaryngology, Aichi Medical University, Aichi, Japan; ${ }^{2}$ Department of Cardiology, Nagoya University Graduate School of Medicine, Nagoya, Japan; ${ }^{3}$ Department of Molecular Cardiology, Nagoya University Graduate School of Medicine, Nagoya, Japan; ${ }^{4}$ Department of Cardiology, Chunichi Hospital, Nagoya, Japan and ${ }^{5}$ Department of Biomedical Informatics, Osaka University Graduate School of Medicine, Osaka, Japan

*Corresponding authors: R Shibata or T Tanigawa, Department of Cardiology, Nagoya University Graduate School of Medicine, 65 Tsurumai, Showa, Nagoya 466-8550, Japan. Tel: + 8152744 2147; Fax: +81 52744 2138; E-mail: rshibata@med.nagoya-u.ac.jp or tanigawa@aichi-med-u.ac.jp

Keywords: adiponectin; age-related hearing impairment; stria vascularis; apoptosis

Abbreviations: ABR, auditory brainstem response; Ad, adenovirus; AMPK, AMP-activated protein kinase; APN, adiponectin; ARHI, age-related hearing impairment; BMI, body mass index; CBF, cochlear blood flow; HUVECs, human umbilical vein endothelial cells; IHC, inner hair cell; KO, knockout; OHC, outer hair cell; PBS, phosphate-buffered saline; PTA, pure tone average; SEM, scanning electron microscopy; SV, stria vascularis; TUNEL, terminal deoxynucleotidyl transferase dUTP nick end labeling; WT, wild type; $\beta$-gal, $\beta$-galactosidase

Received 26.11.13; revised 06.2.14; accepted 24.2.14; Edited by M Federici
} 


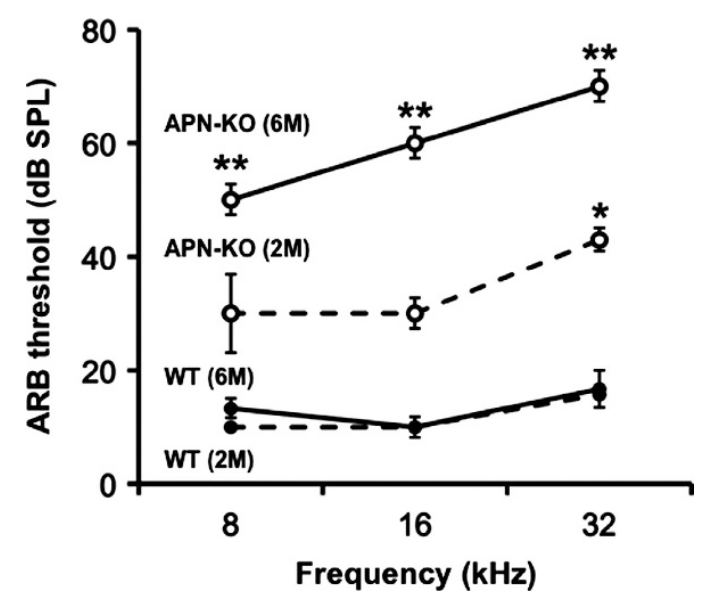

Figure 1 Hearing impairment in APN-KO mice has an early onset and a progressive course. $A B R$ hearing thresholds were measured at 8,16 , and $32 \mathrm{kHz}$, in WT and APN-KO mice at 2 months and 6 months of age $(n=5-7)$. At 2 months and at 6 months of age, WT mice did not show any threshold shifts at any frequencies. At 2 months of age, APN-KO mice exhibited a slight increase in $A B R$ thresholds at high frequency $(32 \mathrm{kHz})$ as compared with WT mice $\left({ }^{*} P<0.01\right)$. At 6 months of age, APN-KO mice showed significantly increased ( $\left.{ }^{\star *} P<0.01\right)$ ABR thresholds at 8,16 , and $32 \mathrm{kHz}$, as compared with WT mice at the ages of both 2 months and 6 months, and with APN-KO mice at the age of 2 months. This change in APN-KO mice at the age of 6 months was greatest at the high frequency tested $(32 \mathrm{kHz})$

(statistically non-significant) increases in ABR thresholds at $8 \mathrm{kHz}$ and $16 \mathrm{kHz}$. At 6 months of age, APN-KO mice exhibited significant increases in ABR thresholds at 8, 16, and $32 \mathrm{kHz}$ compared with WT mice at the ages of 2 months and 6 months, and APN-KO mice at the age of 2 months $(P<0.01)$. Thus, APN-KO mice exhibit an early onset and progressive course of hearing impairment.

Reduced cochlear blood flow and capillary density of the SV in APN-KO mice. Because reduction of blood flow in the cochlea contributes to the progression of hearing impairment, we measured cochlear blood flow (CBF) using a laser-Doppler flowmeter. ${ }^{20,21}$ Quantitative analysis revealed that, at 2 months of age, APN-KO mice showed a marked reduction in CBF $(42.1 \% \pm 4.4 \%)$ as compared with WT mice (Figure 2a, $n=3, P<0.05$ ).

To investigate the extent of cochlear vessel formation at the microcirculatory level, capillary density was measured in histological sections from the SV of cochleae. Figure $2 \mathrm{~b}$ shows representative photomicrographs of tissue immunostained for CD31 (arrows: capillaries in the SV). Quantitative analysis revealed that, at 2 months of age, the capillary density in the SV was significantly reduced in APN-KO mice as compared with WT mice (Figure $2 \mathrm{~b}$, right panel, $n=4, P<0.05$ ).

APN deficiency promotes apoptosis in the organ of Corti. Apoptosis is responsible for the damage to the organ of Corti caused by hypoxia. ${ }^{20,22}$ We performed TUNEL staining in the organs of Corti from WT and APN-KO mice at 2 months of age. Representative photographs of TUNEL-positive (green) nuclei in the organs of Corti at the basal turn are shown in Figure 3a. Higher proportions of TUNEL-positive hair cells were evident at the basal, middle,
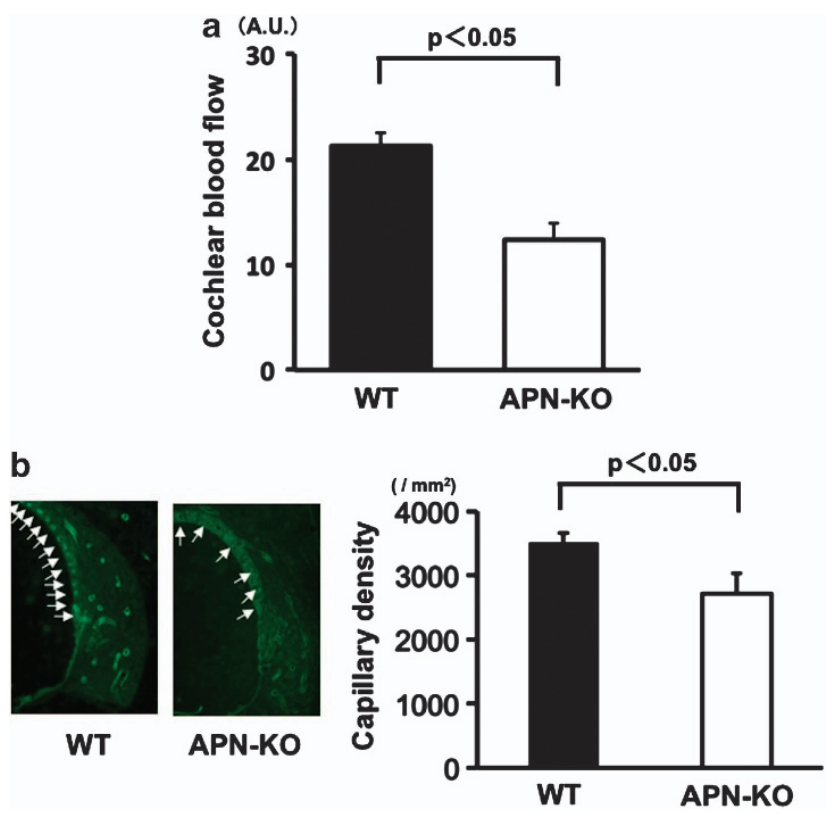

Figure 2 Reduced CBF and capillary density of the SV in APN-KO mice. (a) Reduced CBF in the cochlear basal turn in APN-KO mice. Quantitative analyses of $\mathrm{CBF}$ ratio in APN-KO and WT mice are shown. At 2 months of age, a marked reduction (48.2 $\pm 4.5 \%$ ) was observed in APN-KO mice as compared with WT mice $(P<0.05)$. (b) Reduced capillary density of the SV in the cochlear basal turn in APN-KO mice. (Left panel) Fluorescence staining of the SV with an anti-CD31 monoclonal antibody (green) at 2 months of age, in WT and APN-KO mice (arrows: capillaries in the SV). (Right panel) Quantitative analysis of capillary density in APNKO and WT mice at 2 months of age ( $n=4$ per group). Capillary density in the SV was significantly reduced in APN-KO mice compared with WT mice at 2 months of age $(P<0.05)$

and apical turns of the organ of Corti in APN-KO mice as compared with WT mice. Furthermore, we assessed the effect of APN on caspase-3 activation in the organs of Corti from WT and APN-KO mice at 2 months of age by western blot analysis. The expression level of cleaved caspase-3 was greater in APN-KO mice than in WT mice (Figure 3b).

To examine the effect of APN on apoptosis at the cellular level, auditory $\mathrm{HEI}-\mathrm{OC} 1$ cells were subjected to hypoxia under conditions of serum deprivation in the presence of recombinant human APN or vehicle alone. Treatment with a physiological concentration of APN $(30 \mu \mathrm{g} / \mathrm{ml})$ significantly reduced the frequency of TUNEL-positive cells under hypoxic conditions (Figures 3c, $P<0.001$ ). APN treatment also reduced the frequency of TUNEL-positive human umbilical vein endothelial cells (HUVECs), as previously described $(P<0.01){ }^{23}$

APN can promote apoptosis through modulation of AMP-activated protein kinase (AMPK) activity in various cell types. ${ }^{23,24}$ It has also been reported that AMPK deficiency increases noise vulnerability after acoustic overstimulation. ${ }^{25}$ Thus, we investigated the possible participation of AMPK in the protective actions of APN on hypoxia-induced apoptosis in auditory $\mathrm{HEI}-\mathrm{OC} 1$ cells (Figure $3 \mathrm{~d}$ ). Treatment of HEI-OC1 cells with APN stimulated the phosphorylation of AMPK. Pretreatment with the AMPK inhibitor 'compound C' effectively suppressed the inhibitory effects of APN on apoptosis under hypoxic conditions (Figure $3 e$ ). 
a

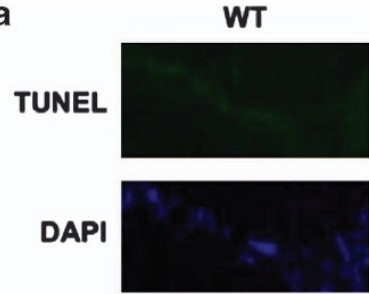

C

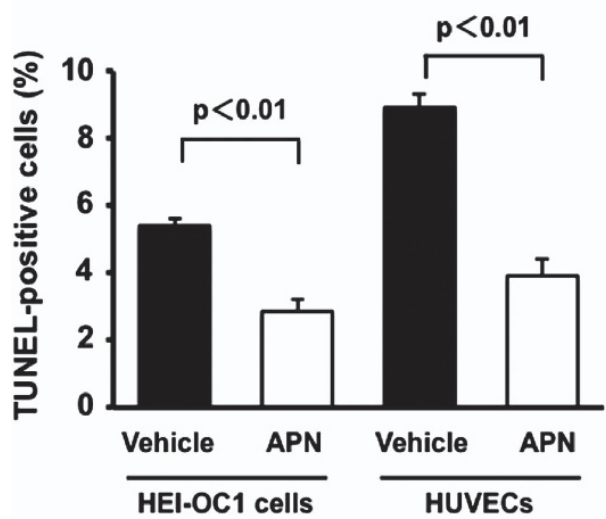

APN-KO
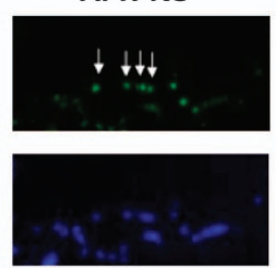

d b

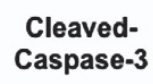

Caspase-3

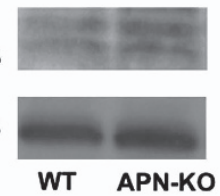

P-AMPK

AMPK

$$
\frac{\text { Vehicle APN }}{\text { HEI-OC1 cells }}
$$

e $\quad p<0.01$
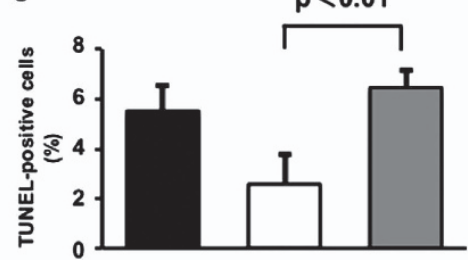

APN

$-$ f

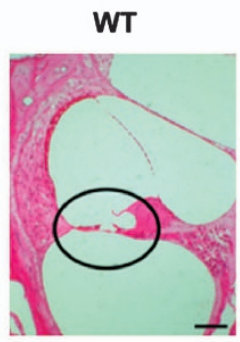

APN-KO

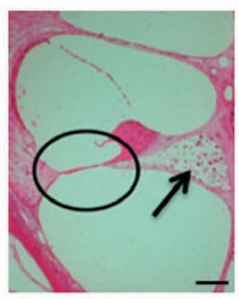

Bar: $50 \mu \mathrm{m}$ g

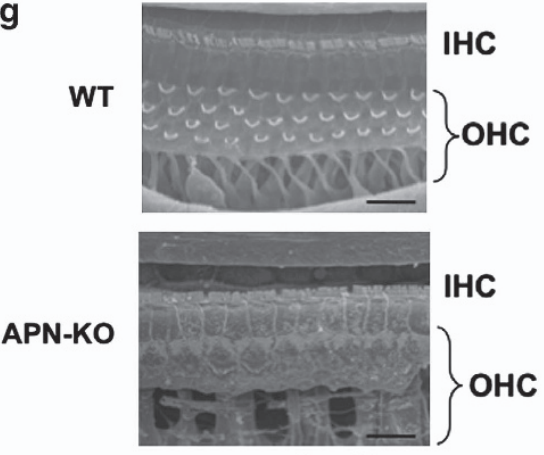

Bar : $20 \mu \mathrm{m}$

Figure 3 APN deficiency promotes apoptosis in the organ of Corti. (a) Representative photographs of TUNEL-positive (green) nuclei in the organs of Corti from WT and APN-KO mice at 2 months of age (arrows: hair cells) ( $n=4$ per group). (b) The degree of cleaved caspase- 3 in the organ of Corti was greater in APN-KO than WT mice at 2 months of age ( $n=4$ per group). (c) The effect of APN on apoptosis at the cellular level. Auditory HEI-OC1 cells were subjected to hypoxia for $24 \mathrm{~h}$ under conditions of serum deprivation, in the presence of recombinant human APN protein or vehicle alone. Treatment with a physiological concentration of APN protein $(30 \mu \mathrm{g} / \mathrm{ml})$ significantly reduced the frequency of TUNEL-positive cells under hypoxic conditions $(P<0.001)$. APN treatment also reduced the frequency of TUNEL-positive cells in HUVECs $(P<0.01)$. (d) APN stimulates the phosphorylation of AMPK in HEI-OC1 cells. The phosphorylation of AMPK (P-AMPK) was determined by western blot analysis. Representative blots are shown from four independent experiments. (e) The effect of an AMPK inhibitor on the anti-apoptotic action of APN. HEI-OC1 cells were pretreated with compound C $(10 \mu \mathrm{m})$ or vehicle. After treatment with compound C, HEl-OC1 cells were cultured in the presence of APN $(30 \mu \mathrm{g} / \mathrm{ml})$ or vehicle under conditions of hypoxia, and TUNELpositive nuclei were quantified ( $n=4$ per group). (f) Representative photomicrographs of the organ of Corti (encircled) in basal turns from WT and APN-KO mice at 6 months old (scale bar: $50 \mu \mathrm{m}$ ). Hair cells were lost from the basal turn of the cochlea in APN-KO mice, whereas in WT mice they were preserved. APN-KO mice also showed severe damage in the regions of spiral ganglion neurons, compared with WT mice (arrow). (g) At 6 months of age, scanning electron microscopy showed that in WT mice, three rows of OHCs and one row of IHCs were found in the organ of Corti at the basal turn of the cochlea (Figure 3d, upper panel, scale bar: $20 \mu \mathrm{m}$ ). However, the OHCs in the basal turn of the cochlea were lost in patches in APN-KO mice of the same age (Figure 3d, lower panel)

Hair cell loss at the organ of Corti in aged APN-KO mice. Because at 2 months of age the extent of apoptosis was higher in the organs of Corti in APN-KO mice than in those of WT mice, we assessed morphological alterations to the organs of Corti in aged WT and APN-KO mice. Cochlear tissue in the basal turn was stained with hematoxylin and eosin in WT and APN-KO mice at 6 months of age. Hair cells were lost at the basal turn of the cochlea in APN-KO mice, whereas hair cells in WT mice were preserved (Figure 3f, encircled). APN-KO mice also showed severe damage in the regions of spiral ganglion neurons, compared with WT mice (arrow).

To further compare the organs of Corti in aged APN-KO and WT mice, we examined them using scanning electron microscopy (SEM). At 6 months of age, in WT mice three rows of outer hair cells (OHCs) and one row of inner hair cells (IHCs) were present in the organ of Corti at the basal turn of the cochlea (Figure $3 \mathrm{~g}$, upper panel). 
However, in APN-KO mice, at 6 months of age, the OHCs in the basal turn of the cochlea were lost in patches (Figure $3 \mathrm{~g}$, lower panel).

APN supplementation prevents hearing impairment in APN-KO mice. To test whether exogenous APN supplementation prevented the development of hearing impairment, we administered adenoviral vectors via the jugular vein, expressing APN (Ad-APN) or $\beta$-galactosidase (Ad- $\beta$-gal) as a control, to WT and APN-KO mice aged 1.5 months. We then performed $A B R$ measurements in these mice at 2 months of age. On the sixth day after injection, plasma APN levels were $11.3 \pm 1.9 \mu \mathrm{g} / \mathrm{ml}$ in WT/Ad- $\beta$-gal, $<0.05 \mu \mathrm{g} / \mathrm{ml}$ in APN-KO/Ad- $\beta$-gal, and $10.5 \pm 1.6 \mu \mathrm{g} / \mathrm{ml}$ in APN-KO/Ad-APN. Ad- $\beta$-gal-treated APN-KO mice exhibited an increase in ABR threshold at $32 \mathrm{kHz}$ compared with Ad- $\beta$-gal-treated WT mice (Figure $4 \mathrm{a}, n=4, P<0.01$ ). Supplementation with APN in APN-KO mice rescued the hearing impairment that was seen in the Ad- $\beta$-gal-treated APN-KO mice $(n=4, P<0.05)$. We also assessed CBF, capillary density, and apoptosis in the organ of Corti. Supplementation with APN in APN-KO mice increased CBF and the capillary density of the SV, and decreased the frequency of TUNEL-positive cells in the organ of Corti (Figures $4 b-d$ ).

Association of APN with hearing threshold level in apparently healthy men. Finally, we examined whether circulating APN level is associated with hearing threshold in apparently healthy Japanese men. Clinical characteristics of the study population are shown in Table 1. Mean BMI, blood
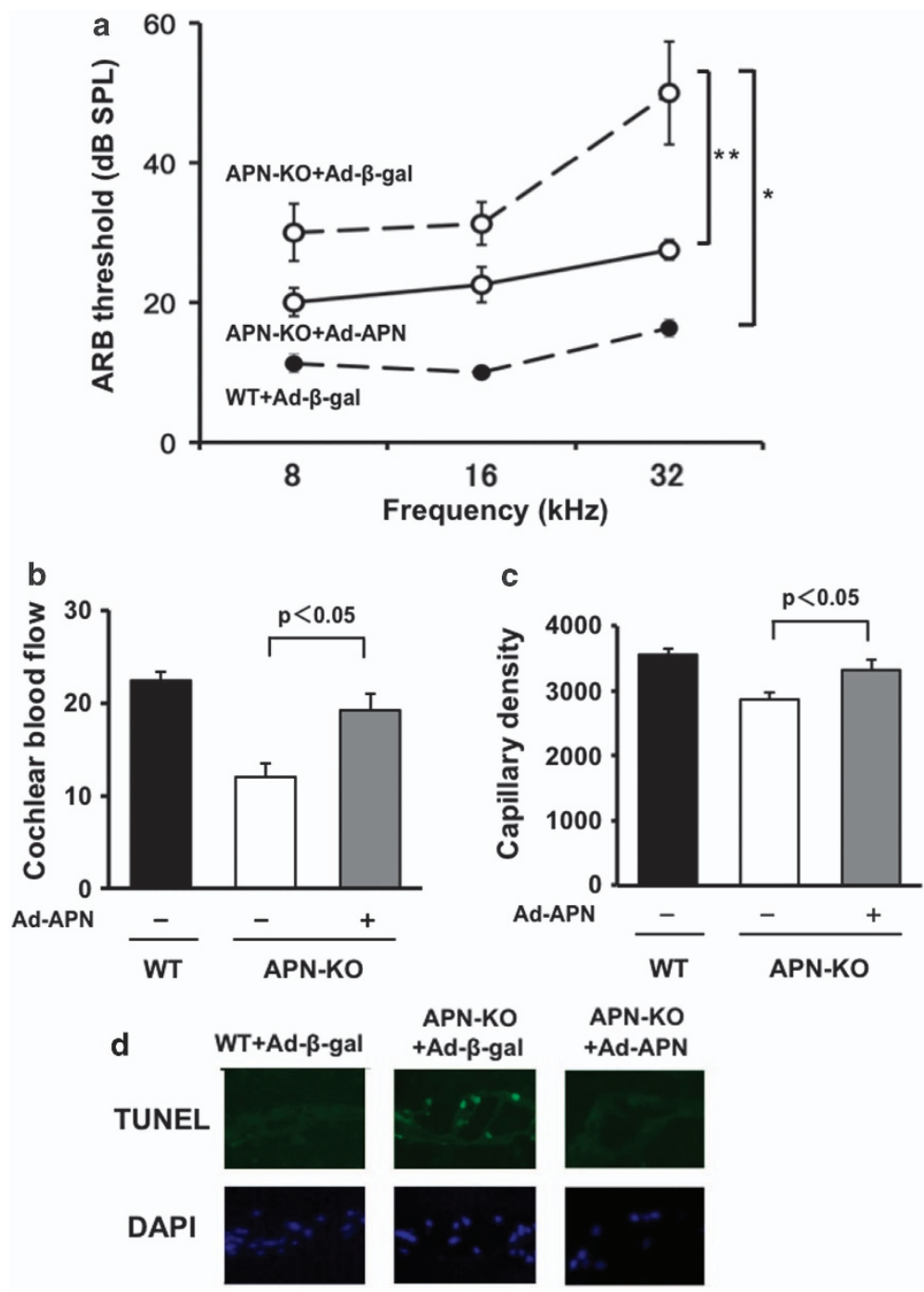

Figure 4 APN supplementation prevents hearing impairment in APN-KO mice. (a) Supplementation with APN could rescue the hearing impairment that was seen in Ad- $\beta$-gal-treated APN-KO mice $\left(n=4,{ }^{*} P<0.01\right)$. At the age of 2 months, Ad- $\beta$-gal-treated APN-KO mice exhibited an increase in ABR thresholds at $32 \mathrm{kHz}$ as compared with Ad- $\beta$-gal-treated WT mice of the same age $\left(n=4,{ }^{\star *} P<0.05\right)$. (b) Supplementation of APN in APN-KO mice increased CBF. Quantitative analyses of CBF ratio are shown ( $n=4$ per group). (c) Ad-APN in APN-KO mice increased the capillary density of the SV. Quantitative analyses of the capillary density in the SV are shown ( $n=4$ per group). (d) Ad-APN in APN-KO mice decreased the frequency of TUNEL-positive cells in the organ of Corti. Representative photographs of TUNEL-positive (green) nuclei in the organ of Corti are shown ( $n=4$ per group) 
pressure, hemoglobin A1c, total cholesterol, LDL cholesterol, $\mathrm{HDL}$ cholesterol, triglycerides, and creatinine levels were within the normal range. Pure tone average (PTA)-low and PTA-high were $19.4 \pm 0.83 \mathrm{~dB}$ and $26.8 \pm 1.17 \mathrm{~dB}$, respectively. The average of the Log-APN levels was $0.64 \pm 0.02 \mu \mathrm{g} / \mathrm{ml}$. Circulating APN levels correlated negatively with $\mathrm{BMI}$, waist circumference, fasting glucose, and triglycerides and positively with HDL cholesterol (Table 1).

Plasma APN levels in hearing-impaired subjects (PTA-high $>25 \mathrm{~dB}$ ) were significantly lower than those in normal-hearing subjects (PTA-high $\leq 25 \mathrm{~dB}$ ) in the high-frequency range (Log-APN: $0.601 \pm 0.025$ versus $0.670 \pm 0.021 \mu \mathrm{g} / \mathrm{ml}, P<0.05)$. In contrast, APN levels did not differ significantly between the two groups in the low-frequency range (Log-APN: $0.604 \pm 0.038$ versus $0.650 \pm 0.018 \mu \mathrm{g} / \mathrm{ml})$. Results of logistic regression analysis

Table 1 Clinical parameters and their associations with APN levels.

\begin{tabular}{lcrr}
\hline Parameters & Subjects $(\boldsymbol{n}=\mathbf{2 0 5})$ & $\boldsymbol{r}$ & $\boldsymbol{P}$-value \\
\hline Age (years) & $53.0 \pm 0.62$ & -0.011 & 0.868 \\
BMl (kg/m $\left.{ }^{2}\right)$ & $23.4 \pm 0.18$ & -0.241 & $<\mathbf{0 . 0 0 1}$ \\
Waist circumference (cm) & $84.8 \pm 0.46$ & -0.231 & $<\mathbf{0 . 0 0 1}$ \\
Systolic BP (mm Hg) & $116.1 \pm 1.2$ & -0.068 & 0.333 \\
Diastolic BP (mm Hg) & $72.9 \pm 0.85$ & -0.072 & 0.306 \\
Fasting glucose (mmol/l) & $103.8 \pm 0.88$ & -0.171 & $\mathbf{0 . 0 1 4}$ \\
Hemoglobin A1c (\%) & $5.48 \pm 0.04$ & -0.044 & 0.531 \\
Total cholesterol (mg/dl) & $203.6 \pm 2.5$ & -0.174 & 0.307 \\
LDL cholesterol (mg/dl) & $3.22 \pm 0.81$ & 0.004 & 0.951 \\
HDL cholesterol (mg/dl) & $54.4 \pm 0.95$ & 0.224 & $\mathbf{0 . 0 0 1}$ \\
Triglycerides (mg/dl) & $139.2 \pm 5.25$ & -0.174 & $\mathbf{0 . 0 1 2}$ \\
Creatinine (mg/dl) & $0.86 \pm 0.01$ & -0.156 & 0.026 \\
Log APN $(\mu \mathrm{g} / \mathrm{ml})$ & $0.64 \pm 0.02$ & & \\
PTA-low & $19.4 \pm 0.83$ & -0.039 & 0.578 \\
PTA-low $>25 \mathrm{~dB}(\%)$ & 19.0 & & 0.258 \\
PTA-high & $26.8 \pm 1.17$ & -0.073 & 0.302 \\
PTA-high $>25 \mathrm{~dB}(\%)$ & 41.4 & & $\mathbf{0 . 0 4 2}$ \\
\end{tabular}

Abbreviations: BMI, body mass index; BP, blood pressure; HDL, high-density lipoprotein; LDL, low-density lipoprotein; PTA, pure tone average. Statistically significant results $(P<0.05)$ are shown in bold. for hearing threshold are shown in Table 2. In single logistic regression analysis, age, fasting glucose, and triglycerides were significantly associated with PTA-low. In multiple logistic regression analysis incorporating age, fasting glucose, and triglycerides, age was significantly correlated with PTA-low. In single regression analysis for PTA-high, age and APN levels were correlated with PTA-high. In multiple regression analysis incorporating age and APN levels, both were significantly associated with PTA-high. Thus, low levels of APN are closely linked with the presence of $\mathrm{ARHI}$, particularly high-frequency sensorineural hearing loss.

\section{Discussion}

The present study provides the first evidence that APN deficiency contributes to ARHI. APN-KO mice showed exacerbated hearing impairment, whereas exogenous APN could reduce hearing impairment in these mice. Furthermore, plasma levels of APN, together with age, are independently associated with ARHI in apparently healthy subjects. Thus, these data suggest that approaches aimed at normalizing circulating APN levels could be valuable for the prevention of hearing impairment.

Accumulating evidence indicates that APN has favorable effects on vascular responses and endothelial cell functions. ${ }^{17,26}$ APN-KO mice exhibit impaired angiogenesis in response to hindlimb ischemia, whereas overexpression of APN promotes ischemia-mediated angiogenesis. ${ }^{17}$ APN supplementation has also been shown to stimulate blood vessel growth in both rabbit corneal angiogenesis and mouse Matrigel plug implantation models. ${ }^{26}$ The present study showed that APN deficiency leads to decreased CBF and capillary density of the SV. Recently, it was reported that hypoxia/ischemia in the cochlea causes damage to the organ of Corti and induces hearing loss. ${ }^{20,21}$ It has also been reported that reduced $\mathrm{CBF}$ and impaired hearing are observed in diabetic mice exposed to loud noise, and ApoE-KO mice fed a high-fat diet. ${ }^{9,27}$ Thus, it is conceivable

Table 2 Correlation with hearing impairment

\begin{tabular}{|c|c|c|c|c|c|c|c|c|}
\hline & \multicolumn{4}{|c|}{ PTA-low > 25 dB } & \multicolumn{4}{|c|}{ PTA-high $>25 \mathrm{~dB}$} \\
\hline & \multicolumn{2}{|l|}{ Single } & \multicolumn{2}{|c|}{ Multiple } & \multicolumn{2}{|l|}{ Single } & \multicolumn{2}{|c|}{ Multiple } \\
\hline & OR $(95 \% \mathrm{Cl})$ & $P$-value & OR $(95 \% \mathrm{Cl})$ & $P$-value & OR $(95 \% \mathrm{Cl})$ & $P$-value & OR $(95 \% \mathrm{Cl})$ & $P$-value \\
\hline Age & 1.08 (1.03-1.13) & 0.001 & 1.08 (1.03-1.13) & $<0.001$ & 1.08 (1.03-1.13) & 0.001 & $1.14(1.09-1.20)$ & $<0.001$ \\
\hline BMI & $1.08(0.95-1.24)$ & 0.252 & & & $1.01(0.91-1.13)$ & 0.807 & & \\
\hline Waist circumference & $1.05(1.00-1.11)$ & 0.064 & & & 1.03 (0.98-1.07) & 0.216 & & \\
\hline Systolic BP & $1.00(0.98-1.02)$ & 0.968 & & & $1.01(0.99-1.02)$ & 0.370 & & \\
\hline Diastolic BP & $1.00(0.97-1.03)$ & 0.974 & & & $1.01(0.98-1.03)$ & 0.633 & & \\
\hline Fasting glucose & $1.03(1.00-1.06)$ & 0.022 & 1.02 (0.99-1.05) & 0.097 & $1.01(0.99-1.03)$ & 0.422 & & \\
\hline Hemoglobin A1c & $1.70(0.92-3.13)$ & 0.083 & & & $0.94(0.55-1.57)$ & 0.803 & & \\
\hline Total cholesterol & $1.00(1.00-1.02)$ & 0.217 & & & $1.00(0.99-1.01)$ & 0.727 & & \\
\hline LDL cholesterol & $1.01(0.99-1.02)$ & 0.259 & & & $1.00(0.99-1.01)$ & 0.531 & & \\
\hline HDL cholesterol & $1.00(0.97-1.02)$ & 0.940 & & & $1.00(0.97-1.02)$ & 0.649 & & \\
\hline Triglyceride & 1.01 (1.00-1.01) & 0.031 & $1.00(1.00-1.01)$ & 0.055 & $1.00(1.00-1.01)$ & 0.597 & & \\
\hline Creatinine & $1.65(0.12-20.9)$ & 0.702 & & & $4.41(0.55-37.0)$ & 0.164 & & \\
\hline Log APN & $0.43(0.10-1.88)$ & 0.258 & & & $0.28(0.08-0.94)$ & 0.042 & $0.21(0.05-0.81)$ & 0.027 \\
\hline
\end{tabular}

Abbreviations: APN, adiponectin; BMI, body mass index; BP, blood pressure; Cl, confidence intervals; HDL, high-density lipoprotein; LDL, low-density lipoprotein; OR, odds ratios; PTA, pure tone average. Multiple regression model includes all variable at baseline with $P<0.05$ by single analysis. Statistically significant results $(P<0.05)$ are shown in bold. 
that loss of APN leads to hypo-perfusion and subsequent hypoxia/ischemia in the cochlea, thereby contributing to the progression of hearing impairment.

It has been suggested that apoptosis of hair cells caused by hypoxia is a key feature of various forms of hearing impairment. $^{20,22}$ In the present study, APN-KO mice showed increased apoptotic activity and loss of hair cells at the organ of Corti. APN also suppressed hypoxia-induced apoptotic activity in cultured auditory HEI-OC1 cells in vitro. Similarly, APN deficiency reportedly causes enhanced apoptosis in the heart following ischemia reperfusion. ${ }^{24,28}$ APN prevents hypoxia-induced apoptotic activity in cardiomyocytes. ${ }^{24}$ Moreover, APN suppresses apoptosis of endothelial cells under conditions of serum starvation. ${ }^{23}$ These data suggest that APN prevents the development of hearing impairment, in part, via suppression of the apoptotic activities of endothelial and auditory sensory hair cells in the cochlea.

Collective evidence from numerous clinical studies indicates that low levels of circulating APN are associated with increased prevalence of obesity-linked diseases, including atherosclerosis and ischemic heart disease. ${ }^{14,15}$ Plasma APN levels in apparently healthy subjects are positively correlated with age. However, this correlation does not remain significant when adjusting for obesity-related risk factors. ${ }^{29,30}$ A previous study showed a negative association between plasma APN concentrations and peripheral hearing thresholds in the Taiwanese population. ${ }^{31}$ That study evaluated both genders and subjects between the ages of 40 and 86 , including healthy adults and subjects with chronic conditions such as heart disease, kidney dysfunction, and diabetes. The present study showed that in apparently healthy middle-aged Japanese men, reduced levels of APN are independently associated with $\mathrm{ARHI}$ after adjustment for age. These data suggest that APN levels are predictive of the progression of ARHI. Thus, APN may represent a biomarker not only for metabolic and vascular disorders, but also for ARHI.

This study has several limitations. First, we only investigated whether APN affects early sensorineural hearing loss using male mice and assessed its clinical significance in ARHI in apparently healthy males. In our preliminary investigation, female APN-KO mice also developed exacerbation of hearing impairment, particularly in the high-frequency range, compared with female WT mice (data not shown). A previous study also reported a negative association between plasma APN concentrations and peripheral hearing thresholds in males and females in the Taiwanese population. ${ }^{31}$ Thus, we believe that disruption of APN results in an early onset and a progressive course of hearing impairment, regardless of gender. Second, we only explored anti-apoptotic effects with regard to the protective action of APN against ARHI. It has been reported that mechanisms contributing to $A R H I$ include hypoxia-induced apoptosis, oxidative stress, and inflammation. $^{13,32,33}$ As well as pro-survival effects, APN exerts several other biological effects in various cell types, including anti-inflammatory and anti-oxidant activities. ${ }^{15}$ Therefore, future experimental studies will be required to determine the APN-mediated intracellular signaling pathways in auditory organs, to gain a better understanding of its protective modes of action.
In conclusion, we found that disruption of APN results in reduced blood flow and increased apoptosis in the cochlea, thereby leading to ARHI. Furthermore, we demonstrated that circulating APN levels are reduced in subjects with ARHI. Collectively, these data suggest that APN represents a target molecule for the prevention of ARHI.

\section{Materials and Methods}

Materials. Recombinant human APN protein generated by a mammalian cell expression system was purchased from BioVendor (Asheville, NC, USA). Adenovirus vectors containing the genes for $\beta$-galactosidase (Ad- $\beta$-gal) and fulllength mouse APN (Ad-APN) were prepared as described previously. ${ }^{34}$ Compound $\mathrm{C}$ was purchased from Calbiochem (San Diego, CA, USA). Primary antibodies specific for phospho-AMPK (Thr-172), pan- $\alpha$-AMPK, and cleaved caspase-3 (Asp175) were purchased from Cell Signaling Technology (Danvers, MA, USA). HEI-OC1 cells were a generous gift from the House research institute (Dr. Kalinec F).

Animals. WT and APN-KO male mice on a C57BL/6 background were used for this study. ${ }^{16}$ Study protocols were approved by the Institutional Animal Care and Use Committee of Nagoya University.

ABR measurement. At the ages of 2 months and 6 months, mice were subjected to $A B R$ measurement under anesthesia with ketamine $(100 \mathrm{mg} / \mathrm{kg})$ and xylazine $(9 \mathrm{mg} / \mathrm{kg})$ as previously described ${ }^{35}$ In brief, mice were placed inside a quiet room, and generation of acoustic stimuli and subsequent recording of evoked potentials were performed using a data acquisition system (AD Instruments, Bella Vista, NSW, Australia). Acoustic stimuli consisting of tone bursts $(0.1 \mathrm{~ms}$ cos2 rise/fall and $1 \mathrm{~ms}$ plateau) were delivered monaurally through a magnetic speaker (CF1, Tucker-Davis Technologies, Alachua, FL, USA) connected to a funnel fitted into the external auditory meatus. To record bioelectrical potentials, subdermal stainless steel needle electrodes were inserted at the vertex (ground), ventrolateral to the measured ear (active), and contralateral to the measured ear. Stimuli were calibrated against a 1/4-inch condenser microphone (UC-54, RION, Tokyo, Japan) connected to a measuring amplifier (NA-42, RION, Tokyo, Japan). Responses between the vertex and mastoid subcutaneous electrodes were amplified with a differential extracellular amplifier (ER-1, Cygnus Technology, Delaware Water Gap, PA, USA). Thresholds were determined for frequencies of 8 , 16 , and $32 \mathrm{kHz}$ from a set of responses at varying intensities with $5-\mathrm{dB} \mathrm{SPL}$ intervals, and electrical signals were averaged at 512 repetitions. In cases of scaleout, thresholds were defined as the maximum intensity of the tone burst stimuli plus $5 \mathrm{~dB}$ SPL, for statistical analysis. In some experiments, $2 \times 10^{8}$ plaque-forming units of Ad-APN or Ad- $\beta$-gal were systemically injected into the tail vein of WT and APN$\mathrm{KO}$ mice at the age of 1.5 months, and ABR measurements were performed.

Analysis of CBF. CBF was measured in WT and APN-KO mice aged 2 months, using a laser-Doppler flowmeter (OMEGA FLOW, FLO-N1, Neuroscience, Tokyo, Japan), as previously described. ${ }^{36,37}$ The left bulla of each mouse was identified and opened carefully using a ventrolateral approach. After removing the mucosa and periosteum overlying the bone, a probe (CS-N type) from a laserDoppler flowmeter was placed over the basal turn of the cochlea, where the maximum output of $\mathrm{CBF}$ was measured in arbitrary units $(\mathrm{AU})$ ). $\mathrm{CBF}$ values (mean for $20 \mathrm{~s}$ after stabilization) were collected using a computer-based chart-recorder.

Histology. Mice were perfused intracardially with physiological saline, followed by $4 \%$ paraformaldehyde in $0.01 \mathrm{M}$ phosphate-buffered saline (PBS) at pH 7.4. Excised temporal bones were immersed in the same fixative at $4{ }^{\circ} \mathrm{C}$ for $4 \mathrm{~h}$. The cochleae were dissected from the temporal bones in PBS. Cochlear specimens were then placed into $0.1 \mathrm{M}$ ethylenediaminetetraacetic acid in PBS for decalcification. The samples were incubated with $30 \%$ sucrose in PBS at $4{ }^{\circ} \mathrm{C}$ before embedding in an OCT compound (Tissue-Tek, Sakura Finetechnical, Tokyo, Japan) and frozen at $-80^{\circ} \mathrm{C}$. Mid-modiolus sections ( $7 \mu \mathrm{m}$ in thickness) were sliced and stained with hematoxylin and eosin. ${ }^{35}$

To determine the capillary density in the SV, we stained tissue sections with an anti-CD31 antibody (Becton Dickinson, Franklin Lakes, NJ, USA). Capillary endothelial cells were quantified by measuring the number of CD31-positive capillaries per $\mathrm{mm}^{2}$ of $\mathrm{SV}$ (capillary density $\left(\right.$ per $\mathrm{mm}^{2}$ ) = number of capillaries/area of SV $\left(\mu \mathrm{m}^{2}\right) \times 1000000$ ) from four different sections in each tissue block. ${ }^{22}$ 
TUNEL staining of the cochlear was performed using the In Situ Cell Death detection kit (Roche, Mannheim, Germany) as described previously. ${ }^{24}$ DAPI was used for nuclear staining. Four different sections from each mouse were examined for the presence of TUNEL-positive cells. The number of TUNEL-positive cells and the total cell numbers were analyzed in each cochlea.

Electron microscopy. Mice were perfused intracardially with physiological saline, followed by $2 \%$ glutaraldehyde plus $2 \%$ PFA in $0.1 \mathrm{M}$ cacodylate buffer at pH 7.4. Temporal bones were then dissected out and perfused with the above-described fixing solution via round and oval windows. The cochlea was further post-fixed in $2 \%$ osmium tetroxide and dehydrated in ethanol for electron microscopy. The specimen was freeze-dried and sputter-coated for observation by SEM (S-800, Hitachi, Japan). ${ }^{36}$

Cultures of auditory $\mathrm{HEI}-\mathrm{OC} 1$ cells. HEl-OC1 cells, a murine auditory cell line, were cultured in DMEM containing $10 \%$ heat-inactivated fetal calf serum (GIBCO-BRL, Gaithersburg, MD, USA) and antibiotics at $33^{\circ} \mathrm{C}$ under $5 \% \mathrm{CO}_{2}$ HUVECs were cultured in endothelial cell growth medium-2. ${ }^{26,38,39}$ After $12 \mathrm{~h}$ of serum starvation, $\mathrm{HEI}-\mathrm{OC} 1$ cells and HUVECs were treated with the recombinant APN protein $(30 \mu \mathrm{g} / \mathrm{ml})$ or the vehicle alone, followed by $24 \mathrm{~h}$ under hypoxic conditions. Hypoxic conditions were generated using an AnaeroPack (Mitsubishi GAS Chemical, Tokyo, Japan). TUNEL staining was performed using the In Situ Cell Death detection kit as previously described (Roche). ${ }^{39}$ TUNEL-positive cells were counted in five randomly selected microscope fields. Each experiment was repeated three times.

Western blot analysis. The organ of Corti and cell samples were prepared in lysis buffer containing $1 \mathrm{~mm}$ PMSF (Sigma, St. Louis, MO, USA). The protein concentration was calculated using a BCA protein assay kit (Thermo Scientific, Waltham, MA, USA). Equal amounts of proteins were separated by denaturing SDSPAGE. Proteins were transferred onto PVDF membrane (Bio Rad, Hercules, CA, USA) and probed with the primary antibody followed by incubation with the horseradish peroxidase-conjugated secondary antibody. The ECL Plus (GE Healthcare, Princeton, NJ, USA) system was used for detection of the protein signal.

Clinical study. Participants in this study were apparently healthy subjects who visited Chunichi Hospital in Nagoya for a routine checkup between 2006 and 2009. A total of 205 Japanese men with no history of cardiovascular disease and who were not taking any medication participated in this study. All subjects provided written informed consent, and the study was approved by the ethics committee of Nagoya University School of Medicine and Chunichi Hospital.

All audiometry procedures were conducted in a double-walled room. Airconduction pure-tone thresholds at octave intervals from 125 to $8000 \mathrm{~Hz}$ were obtained using diagnostic audiometers (AA-73A, Rion, Tokyo, Japan). The thresholds over the predetermined output level, according to the Japanese Industrial Standards T 1201 calibration, were treated as that level plus an additional $5 \mathrm{~dB}$. PTA was calculated for the worse ear. The PTA-low was calculated as the average threshold across the 125-, 250-, and 500-Hz thresholds. The PTA-high was the average across the 2000-, 4000-, and $8000-\mathrm{Hz}$ thresholds. Hearing impairment was defined as PTA greater than $25 \mathrm{~dB}$ according to World Health Organization criteria.

Venous blood samples were obtained for chemical analysis after an overnight fast. APN level was determined with the use of a latex turbidometric immunoassay (Otsuka Pharmaceutical Corporation, Tokushima, Japan). Because the distributions of APN were skewed, we used logarithmically transformed values for statistical analysis. Standard assays were used to measure glucose, hemoglobin A1c, insulin, total cholesterol, HDL cholesterol, LDL cholesterol, triglycerides, and creatinine. After an appropriate rest of $10 \mathrm{~min}$, sitting blood pressure was measured. BMI was calculated as the ratio of weight to squared height.

Statistical analysis. All data are presented as mean \pm S.E.M. Statistical analysis was performed using ANOVA followed by the Scheffe's method or Mann-Whitney U-test. In the clinical study, the correlations between APN levels and the indicated parameters were examined by single logistic regression analysis. Single and multiple logistic regression analyses were also performed to analyze the correlations of the indicated parameters with hearing impairment (PTA-low $>25 \mathrm{~dB}$ and PTA-high $>25 \mathrm{~dB}$ ). A $P$-value of $<0.05$ was considered statistically significant. All analyses were performed using JMP (version 6.03; SAS Institute, Tokyo, Japan).

\section{Conflict of Interest}

The authors declare no conflict of interest.

Acknowledgements. This work was supported by a Grant-in-Aid for Young Scientists B, Banyu Life Science Foundation International, Kanae Foundation, and Kowa Life Science Foundation, awarded to R Shibata.

1. Liu XZ, Yan D. Ageing and hearing loss. J Pathol 2007; 211: 188-197.

2. Jerger J, Chmiel R, Wilson N, Luchi R. Hearing impairment in older adults: new concepts. J Am Geriatr Soc 1995; 43: 928-935.

3. Seidman MD, Ahmad N, Bai U. Molecular mechanisms of age-related hearing loss. Ageing Res Rev 2002; 1: 331-343.

4. Kim TS, Park SW, Kim DY, Kim EB, Chung JW, So HS. Visceral adipose tissue is significantly associated with hearing thresholds in adult women. Clin Endocrinol 2013; 80: 368-375.

5. Hwang JH, Wu CC, Hsu CJ, Liu TC, Yang WS. Association of central obesity with the severity and audiometric configurations of age-related hearing impairment. Obesity 2009; 17: 1796-1801.

6. Suzuki K, Kaneko M, Murai K. Influence of serum lipids on auditory function. Laryngoscope 2000; 110(10 Pt 1): 1736-1738

7. Dalton DS, Cruickshanks KJ, Klein R, Klein BE, Wiley TL. Association of NIDDM and hearing loss. Diabet Care 1998: 21: 1540-1544.

8. Cullen JR, Cinnamond MJ. Hearing loss in diabetics. J Laryngol Otol 1993; 107: 179-182.

9. Guo $Y$, Zhang C, Du X, Nair U, Yoo TJ. Morphological and functional alterations of the cochlea in apolipoprotein E gene deficient mice. Hear Res 2005; 208: $54-67$.

10. Vasilyeva ON, Frisina ST, Zhu X, Walton JP, Frisina RD. Interactions of hearing loss and diabetes mellitus in the middle age CBA/CaJ mouse model of presbycusis. Hear Res 2009; 249: 44-53.

11. Ishikawa $T$, Naito $Y$, Taniguchi $K$. Hearing impairment in WBN/Kob rats with spontaneous diabetes mellitus. Diabetologia 1995; 38: 649-655.

12. Someya S, Yamasoba T, Weindruch R, Prolla TA, Tanokura M. Caloric restriction suppresses apoptotic cell death in the mammalian cochlea and leads to prevention of presbycusis. Neurobiol Aging 2007; 28: 1613-1622.

13. Someya S, Yu W, Hallows WC, Xu J, Vann JM, Leeuwenburgh C et al. Sirt3 mediates reduction of oxidative damage and prevention of age-related hearing loss under caloric restriction. Cell 2010; 143: 802-812.

14. Ouchi N, Parker JL, Lugus JJ, Walsh K. Adipokines in inflammation and metabolic disease. Nat Rev Immunol 2011; 11: 85-97.

15. Shibata R, Murohara T, Ouchi N. Protective role of adiponectin in cardiovascular disease. Curr Med Chem 2012; 19: 5459-5466.

16. Maeda N, Shimomura I, Kishida K, Nishizawa H, Matsuda M, Nagaretani $\mathrm{H}$ et al. Diet-induced insulin resistance in mice lacking adiponectin/ACRP30. Nat Med 2002; 8: 731-737.

17. Shibata R, Ouchi N, Kihara S, Sato K, Funahashi T, Walsh K. Adiponectin stimulates angiogenesis in response to tissue ischemia through stimulation of amp-activated protein kinase signaling. J Biol Chem 2004; 279: 28670-28674.

18. Ouchi N, Ohishi M, Kihara S, Funahashi T, Nakamura T, Nagaretani H et al. Association of hypoadiponectinemia with impaired vasoreactivity. Hypertension 2003; 42: $231-234$

19. Okamoto $Y$, Kihara S, Ouchi N, Nishida M, Arita Y, Kumada M et al. Adiponectin reduces atherosclerosis in apolipoprotein E-deficient mice. Circulation 2002; 106: 2767-2770.

20. Cheng AG, Huang T, Stracher A, Kim A, Liu W, Malgrange B et al. Calpain inhibitors protect auditory sensory cells from hypoxia and neurotrophin-withdrawal induced apoptosis. Brain Res 1999; 850: 234-243.

21. Lamm K, Arnold W. Noise-induced cochlear hypoxia is intensity dependent, correlates with hearing loss and precedes reduction of cochlear blood flow. Audiol Neurootol 1996; 1: 148-160.

22. Zhu S, Du X, Cai $Q$, Guo Y, Liu L, Cheng $W$ et al. Impaired stria vascularis in the inner ear of apolipoprotein E gene knockout mice. J Otorhinolaryngol Relat Spec 2008; 70: 373-380.

23. Kobayashi H, Ouchi N, Kihara S, Walsh K, Kumada M, Abe Y et al. Selective suppression of endothelial cell apoptosis by the high molecular weight form of adiponectin. Circ Res 2004; 94: e27-e31.

24. Shibata R, Sato K, Pimentel DR, Takemura Y, Kihara S, Ohashi K et al. Adiponectin protects against myocardial ischemia-reperfusion injury through AMPK- and COX-2dependent mechanisms. Nat Med 2005; 11: 1096-1103.

25. Foller M, Jaumann M, Dettling J, Saxena A, Pakladok T, Munoz C et al. AMP-activated protein kinase in BK-channel regulation and protection against hearing loss following acoustic overstimulation. FASEB J 2012; 26: 4243-4253.

26. Ouchi N, Kobayashi H, Kihara S, Kumada M, Sato K, Inoue T et al. Adiponectin stimulates angiogenesis by promoting cross-talk between AMP-activated protein kinase and Akt signaling in endothelial cells. J Biol Chem 2004; 279: 1304-1309. 
27. Fujita T, Yamashita D, Katsunuma S, Hasegawa S, Tanimoto H, Nibu K. Increased inner ear susceptibility to noise injury in mice with streptozotocin-induced diabetes. Diabetes 2012; 61: 2980-2986.

28. Tao L, Gao E, Jiao X, Yuan Y, Li S, Christopher TA et al. Adiponectin cardioprotection after myocardial ischemia/reperfusion involves the reduction of oxidative/nitrative stress. Circulation 2007; 115: 1408-1416.

29. Miles EA, Rees D, Banerjee T, Cazzola R, Lewis S, Wood R et al. Age-related increases in circulating inflammatory markers in men are independent of $\mathrm{BMI}$, blood pressure and blood lipid concentrations. Atherosclerosis 2008; 196: 298-305.

30. Adamczak M, Rzepka E, Chudek J, Wiecek A. Ageing and plasma adiponectin concentration in apparently healthy males and females. Clin Endocrinol 2005; 62: 114-118.

31. Hwang JH, Hsu CJ, Liu TC, Yang WS. Association of plasma adiponectin levels with hearing thresholds in adults. Clin Endocrinol 2011; 75: 614-620.

32. Hwang JH, Hsu CJ, Yu WH, Liu TC, Yang WS. Diet-induced obesity exacerbates auditory degeneration via hypoxia, inflammation, and apoptosis signaling pathways in $\mathrm{CD} / 1$ mice. PLoS One 2013; 8: e60730.

33. Hwang JH, Chen JC, Yang WS, Liu TC. Waist circumference is associated with pitch pattern sequence score in older male adults. Int $J$ Audiol 2012; 51: 920-925.

34. Shibata R, Ouchi N, Ito M, Kihara S, Shiojima I, Pimentel DR et al. Adiponectin-mediated modulation of hypertrophic signals in the heart. Nat Med 2004; 10: 1384-1389.

35. Shiga A, Nakagawa T, Nakayama M, Endo T, Iguchi F, Kim TS et al. Aging effects on vestibulo-ocular responses in C57BL/6 mice: comparison with alteration in auditory function. Audiol Neuro-otol 2005; 10: 97-104.
36. Iwasaki S, Nagura M, Miyashita H, Umemura K, Hoshino T. Focal damage to cochlear microcirculation measured using a non-contact laser blood flowmeter in guinea pigs. Acta Oto-laryngologica 1998; 118: 666-672.

37. Sone M, Muramatsu H, Muramatsu T, Nakashima T. Morphological observation of the stria vascularis in midkine and pleiotrophin knockout mice. Auris Nasus Larynx 2011; 38: 41-45.

38. Tanigawa T, Morikawa A, Hayashi K, Dan K, Tsuchihashi N, Goto F et al. Auditory cells produce nitric oxide in response to bacterial lipopolysaccharide. Innate Immunity 2013; 19: $115-120$.

39. Maruyama S, Shibata R, Kikuchi R, Izumiya Y, Rokutanda T, Araki S et al. Fat-derived factor omentin stimulates endothelial cell function and ischemia-induced revascularization via endothelial nitric oxide synthase-dependent mechanism. J Biol Chem 2012; 287: 408-417.

(c) (i) $(9)$ Cell Death and Disease is an open-access journal published by Nature Publishing Group. This work is licensed under a Creative Commons Attribution-NonCommercialNoDerivs 3.0 Unported License. The images or other third party material in this article are included in the article's Creative Commons license, unless indicated otherwise in the credit line; if the material is not included under the Creative Commons license, users will need to obtain permission from the license holder to reproduce the material. To view a copy of this license, visit http://creativecommons.org/licenses/ by-nc-nd/3.0/ 